\title{
TURBULENT TRANSPORT AND HEATING IN THE AURORAL PLASMA OF THE TOPSIDE IONOSPHERE
}

\author{
J. A. IONSON \\ Laboratory for Astronomy and Solar Physics, NASA/Goddard Space Flight Center, Greenbelt, MD, 20771, U.S.A. \\ and \\ R. S. B. ONG* and E. G. FONTHEIM \\ Space Physics Research Laboratory, Department of Atmospheric and Oceanic Science, The University of Michigan, \\ Ann Arbor, Michigan 48109, U.S.A.
}

(Received 21 June 1978)

\begin{abstract}
Using plasma parameters from a typical stormtime ionospheric energy balance model, we have investigated the effects of plasma turbulence on the auroral magnetoplasma. The turbulence is assumed to be comprised of electrostatic ion cyclotron waves. These waves have been driven to a nonthermal level by a geomagnetic field-aligned, current-driven instability. The evolution of this instability is shown to proceed in two stages and indicates an anomalous increase in field-aligned electrical resistivity and cross-field ion thermal conductivity as well as a decrease in electron thermal conductivity along the geomagnetic field. In addition, this turbulence heats ions perpendicular to the geomagnetic field and hence leads to a significant ion temperature anisotropy.
\end{abstract}

\section{INTRODUCTION}

Energy balance in the topside ionosphere of the auroral zone is a very important problem, in particular during stormtimes. The few existing observations indicate that the plasma is turbulent with transport properties such as electrical and thermal conductivity differing significantly from those based upon Coulomb collisions (Spitzer, 1962). This has recently been shown by two independent sets of observations (Mozer, 1976; Fontheim et al., 1978). The origin of this turbulence is quite possibly the result of current-driven instabilities. Such currents are part of the well-known Birkeland network and can become quite large during an auroral event (Arnoldy, 1974).

With regard to field-aligned current-driven instabilities (currents being parallel to an ambient magnetic field), a great deal of work has been done concerning the ion acoustic instability (Biskamp and Chodura, 1971; Gary and Paul, 1971; Caponi and Davidson, 1973) and the Buneman instability (Ichimaru, 1975). These particular current-driven instabilities are characterized by large thresholds which require a large relative velocity between the electron and ion fluids. Such thresholds are not exceeded except possibly at very high altitudes in the auroral plasma (perhaps above $7000 \mathrm{~km}$ ). However, the presence of the geomagnetic field in-

* Department of Aerospace Engineering, The University of Michigan, Ann Arbor, Michigan 48109, U.S.A. creases the number of plasma modes that can be excited by essentially eliminating ion Landau damping transverse to the field. As a consequence, electrostatic ion-cyclotron waves can be driven unstable by relatively smaller electron drift velocities (relative velocity of the current-carrying electrons with respect to the stationary ions). As found by Drummond and Rosenbluth (1962), and subsequently applied to the topside ionosphere by Kindel and Kennel (1971), the electrostatic ioncyclotron (IC) instability has the lowest threshold when compared to other current-driven instabilities when $0.01<T_{e} / T_{i}<8$.0. For example, when $T_{e} \simeq T_{i}$ an electron drift velocity $v_{d} \simeq 12 v_{i}$ ( $v_{i}$ being the ion thermal velocity) is sufficient to excite the electrostatic ion-cyclotron instability whereas excitation of an ion-acoustic or Buneman instability would require a drift velocity which is three times larger. For the topside ionosphere a drift of $12 v_{i}$ implies an electron flux of the order of $10^{9}$ electrons $\mathrm{cm}^{-2} \mathrm{~s}$, or equivalently, an electron current density of the order of $1.6 \times 10^{-6} \mathrm{~A}^{-2} \mathrm{~m}$. Such electron fluxes are often exceeded in an auroral region above $1000 \mathrm{~km}$ (Reasoner and Chappell, 1973; Cloutier et al., 1970). Therefore, in this report we will assume that the ionospheric turbulence originates from a current driven electrostatic ioncyclotron instability and investigate the effects of this turbulence, viz. plasma heating and anomalous transport.

In Section 2 we describe the nonlinear theory of 
anomalous transport and heating while in Sections 3 and 4 we discuss the manner in which the electrostatic ion-cyclotron instability saturates. As found by Ionson et al. (1976) saturation occurs in two successive stages. The first stage is completed in a few ion Larmor periods and lasts only seconds, after which a residual second stage level of turbulence becomes established. This second stage then lasts throughout the length of the disturbance. Turbulent heating, electrical resistivity, electron and ion thermal conductivities have been evaluated for both stages and are given as a function of altitude for typical values of the current density.

\section{TRANSPORT AND HEATING PROCESSES IN A TURBULENT MAGNETOPLASMA}

The general role that transport processes play in fluids and plasmas is to restore them to a state of macroscopic equilibrium. In a thermal plasma this equilibrium is a time-independent velocity distribution of electrons and ions which is supported by stochastic particle-particle scattering. A measure of this scattering process in a non-turbulent magnetoplasma is given by the particle-particle collision frequencies which in turn are related to the random thermal fluctuations residing in the plasma. Consequently, collisional transport processes in a thermal plasma scale as $\epsilon_{i h}$, the energy density of the thermal fluctuations normalized to the macroscopic pressure of the plasma. However, when a plasma is turbulent, the transport processes are not necessarily determined by particle-particle scattering. The particle orbits are now stochastically altered by nonthermal fluctuations in the plasma. These fluctuations result from linearly growing unstable wave fields which, in our case, are the consequence of a current driven electrostatic ion-cyclotron instability. Eventually the energy density of these fluctuations saturates nonlinearly at some level $\epsilon_{s}$ (normalized to the thermal pressure). Therefore, transport processes in a turbulent magnetoplasma depend primarily upon $\epsilon_{\mathrm{s}}$ rather than $\epsilon_{\mathrm{th}}$. Furthermore, it is very important to note that, in constrast to classical (i.e. collisional) transport, anomalous transport in a turbulent magnetoplasma is quite selective. That is, it depends crucially upon the wave-particle interactions which allow the system to remain in a turbulent state.

To first order in $\epsilon$ ( $\epsilon$ being the energy density of the turbulent fluctuations normalized to the plasma pressure) the velocity distribution of particles (type $\alpha)$ is described by

$$
\frac{\mathrm{D} f_{\alpha}}{\mathrm{D} t}=\frac{\partial}{\partial v} \cdot \mathbf{D}^{\alpha}(\epsilon) \cdot \frac{\partial f_{\alpha}}{\partial v}
$$

where $\mathrm{D} / \mathrm{D} t$ is the usual convective derivative and where $\mathbf{D}^{\boldsymbol{\alpha}}(\boldsymbol{\epsilon})$ is a velocity space diffusion tensor. This tensor can be cast into non-resonant and resonant parts with resonance meaning that in the particles frame, the turbulent waves are doppler shifted to zero frequency. Therefore, following Weinstock (1970) $\mathbf{D}^{\alpha}(\epsilon)$ can be written as

$$
\begin{aligned}
& \left(\mathrm{D}_{\perp}{ }^{\alpha}, \mathrm{D}_{\perp \|}{ }^{\alpha}, \mathrm{D}_{\|}{ }^{\alpha}\right)_{\text {resonant }}=\omega_{\mathrm{p \alpha}}{ }^{2} v_{\alpha}{ }^{2} \\
& \quad \times \sum_{n} \int \mathrm{d}^{3} \mathbf{k} J_{n}{ }^{2}\left(\lambda_{\alpha}\right) R_{\alpha} \epsilon_{\mathrm{x}}\left(\frac{k_{\perp}{ }^{2} n^{2}}{k^{2} \lambda_{\alpha}{ }^{2}}, \frac{k_{\|} k_{\perp} n}{k^{2} \lambda_{\alpha}}, \frac{k_{\|}{ }^{2}}{k^{2}}\right)
\end{aligned}
$$

and

$$
\begin{aligned}
& \left(\mathrm{D}_{\perp}{ }^{\alpha}, \mathrm{D}_{\perp \|}{ }^{\alpha}, \mathrm{D}_{\|}{ }^{\alpha}\right)_{\text {non-resonant }}=\omega_{\mathrm{p \alpha}}{ }^{2} v_{\alpha}{ }^{2} \\
& \times \sum_{n} \int \mathrm{d}^{3} \mathbf{k} \frac{J_{n}{ }^{2}\left(\lambda_{\alpha}\right) \frac{\partial \epsilon_{\mathbf{k}}}{\partial t}}{\left(|\omega|-k_{\|} v_{\|}-n \Omega_{\alpha}\right)^{2}}\left(\frac{k_{\perp}{ }^{2} n^{2}}{k^{2} \lambda_{\alpha}}, \frac{k_{\|} k_{\perp} n}{k^{2} \lambda_{\alpha}}, \frac{k_{\|}{ }^{2}}{k^{2}}\right)
\end{aligned}
$$

where $J_{n}$ is a Bessel function of order $n, \Omega_{\alpha}=$ $e_{\alpha} B / m_{\alpha} c, \lambda_{\alpha}=k_{\perp} v_{\perp} /\left|\Omega_{\alpha}\right|$ and wherc the integration in equation (3) excludes the pole $\left(|\omega|-k_{\perp} v_{\perp}-\right.$ $n \Omega_{\alpha}=0$. In equations (2) and (3) $\epsilon_{\mathbf{k}}$ represents the normalized energy density of waves with wavenumber $\mathbf{k}$ and the subscripts $\|$ and $\perp$ refer to directions which are parallel and perpendicular to an ambient magnetic field of magnitude $B$. In addition, the term $R_{\alpha}$ found in equation (2) is a resonance function which is defined by

$$
\begin{aligned}
& R_{\alpha}=\mathscr{R} e \int_{0}^{\infty} \mathrm{d} t \exp [ i\left(\omega-k_{\|} v_{\|}-n \Omega_{\alpha}\right) \\
&\left.\times t-\frac{1}{2}\left\langle\left(\mathbf{k} \cdot \delta \mathbf{x}^{\alpha}\right)^{2}\right\rangle\right]
\end{aligned}
$$

The term $\left\langle\left(\mathbf{k} \cdot \delta \mathbf{x}^{\alpha}\right)^{2}\right\rangle$ represents the average mean square deviation from the mean of a particle trajectory in the turbulent field and is given by

$$
\left\langle\left(\mathbf{k} \cdot \delta \mathbf{x}^{\alpha}\right)^{2}\right\rangle=2\left(\mathbf{k} \cdot \mathbf{D}^{* \alpha} \cdot \mathbf{k}\right) t
$$

$\mathrm{D}^{* \alpha}$ appears as a resonant spatial diffusion tensor in equation (33) of Weinstock's (1970) article. Note that a small nonlinear frequency shift has been neglected in $R_{\alpha}$ (Weinstock, 1972). With a sufficiently large magnetic field such that $\Omega_{\alpha} t_{c} \gg 1$ where $t_{c}$ is the correlation time of the electric field fluctuations, $D^{* \alpha}$ can be written as

$$
\begin{aligned}
\left(\mathbf{k} \cdot \mathbf{D}^{* \alpha} \cdot \mathbf{k}\right) t=\frac{1}{3} k_{\|}{ }^{2} \mathrm{D}_{\|}{ }^{\alpha} t^{3}+\frac{k_{\perp} k_{\|}}{\Omega_{\alpha}} \mathrm{D}_{\perp \|}{ }^{\alpha} & t^{2} \\
& +\frac{k_{\perp}{ }^{2}}{\Omega_{\alpha}{ }^{2}} \mathrm{D}_{\perp}{ }^{\alpha} t
\end{aligned}
$$


where it is understood that only the resonant part of $\mathbf{D}^{\alpha}$ (equation (2)) contributes. From equations (5) and (6) the resonance function $R_{\mathrm{u}}$ described by equation (4) can be approximated by

$$
\begin{aligned}
R_{\alpha}=\mathscr{R} e \int_{0}^{\infty} \mathrm{d} T \exp \left[i\left(\omega-k_{\|} v_{\|}-n \Omega_{\alpha}\right)\right. \\
\left.-\left(\left(\Delta \omega_{\|}\right)_{\alpha}+\left(\Delta \omega_{\perp}\right)_{\alpha}\right) T\right]
\end{aligned}
$$

with

and

$$
\left(\Delta \omega_{\|}\right)_{\alpha} \simeq\left(\frac{1}{3} k_{\|}^{2} \mathrm{D}_{\|}^{\alpha}\right)^{1 / 3}
$$

$$
\left(\Delta \omega_{\perp}\right)_{\alpha} \simeq \frac{k_{\perp}{ }^{2}}{\Omega_{\alpha}^{2}} \mathrm{D}_{\perp}^{\alpha} .
$$

Upon saturation of the instability $\partial \epsilon_{k} / \partial t \rightarrow 0$ and it is clear from equation (3) that only the resonant part of the velocity space diffusion tensor is important. Using the general properties of electrostatic ion-cyclotron turbulence, i.e. $k \simeq k_{\perp} \simeq 10 k_{\mathrm{H}}$ for $T_{e} \approx T_{i} \quad$ and $\quad k_{\perp} \rho_{c i} \simeq 1 \quad\left(\rho_{c \alpha}=v_{\alpha} / \Omega_{\alpha} \quad\right.$ with $v_{\alpha}=$ $\left.\left(2 T_{\alpha} / m_{\alpha}\right)^{1 / 2}\right)$, and equation (7) in equation (2) yields

$$
\begin{aligned}
& \mathrm{D}_{\perp}{ }^{e}=\left(\frac{1.3 \pi e^{2}}{m_{e}{ }^{2}}\right)\left(n_{e} T_{e}\right)\left(k_{\|}{ }^{2} / k^{2}\right) \\
& \quad \times\left\{\left(\Delta \omega_{\|}\right)_{e} \epsilon_{s} /\left[\left(\omega-k_{\|} v_{\|}\right)^{2}+\left(\Delta \omega_{\|}\right)_{e}{ }^{2}\right]\right\} \\
& \mathrm{D}_{\perp}{ }^{i} \simeq\left(8 \pi e^{2} / m_{i}{ }^{2}\right)\left(n_{e} T_{e}\right) J_{1}{ }^{2}(1)\left(k_{\perp} / k\right)^{2} \\
& \quad \times\left\{\left(\Delta \omega_{\perp}\right)_{i} \epsilon_{s} /\left[\left(\omega-k_{\|} v_{\|}-\Omega_{i}\right)^{2}+\left(\Delta \omega_{\perp}\right)_{i}{ }^{2}\right]\right\}
\end{aligned}
$$

with all other matrix elements of the velocity space diffusion tensor being small in comparison to equations (10) and (11) and where $\epsilon_{s}$ is the total normalized energy density of the saturated turbulence. In obtaining equations (10) and (11) we have approximated the resonance function $R_{\alpha}$ by a Lorentzian with half width $(\Delta \omega)_{\alpha}=\left(\Delta \omega_{\|}\right)_{\alpha}+\left(\Delta \omega_{\perp}\right)_{\alpha}$ in the following manner:

$$
R_{\alpha}=(\Delta \omega)_{\alpha} /\left[\left(\omega-k_{\|} v_{\|}-n \Omega_{\alpha}\right)^{2}+(\Delta \omega)_{\alpha}{ }^{2}\right]
$$

When $T_{e} \approx T_{i}$ with no temperature anisotropy the wave of maximum growth is such that $k_{\perp} / k_{\|} \simeq 10$ and $\omega-\Omega_{i} \simeq 0.2 \Omega_{i}$ with a threshold (or critical) drift velocity $v_{c}=12 v_{i}$ (Kindel and Kennel, 1971). With these properties and equations (8) and (9), equations (10) and (11) reduce to

$$
\mathrm{D}_{\|}^{e} \simeq 0.01\left(e / m_{e}\right)^{2}\left(n_{e} T_{e} / \Omega_{i}\right)\left(\Omega_{i} / \omega_{p i}\right)^{1 / 2} \epsilon_{s}^{3 / 4}
$$

and

$$
D_{\perp}{ }^{i} \simeq\left(e / m_{i}\right)^{2}\left(n_{e} T_{e} / \Omega_{i}\right) \epsilon_{s} .
$$

These expressions can now be used in determining the anomalous electrical resistivity and thermal conductivity as well as the amount of turbulent heating.

The electrical resistivity is determined by taking the first velocity moment of equation (1). The result is given by

$$
\eta^{\mathrm{IC}} \simeq 0.3\left(\omega_{\mathrm{pi}} / \Omega_{\mathrm{i}}\right)^{1 / 2} \epsilon_{\mathrm{s}}^{3 / 4} \omega_{\mathrm{pe}}^{-1}
$$

where equation (13) has been used. By associating an effective collision frequency $v_{e f f}$ with this resistivity, the electron thermal conductivity $\kappa_{\| e}{ }^{1 C}$ parallel to the magnetic field can be written as

$$
\kappa_{\| k}{ }^{I C}=\left(v_{e e} / v_{e f f}\right) \kappa_{\mathbb{k}}^{\text {classical }}
$$

where $v_{e e}$ is the electron-electron collision frequency and where $v_{\text {eff }}$ is given by

$$
v_{\text {eff }}=\left(\omega_{\mathrm{pu}}^{2} / 4 \pi\right) \eta^{1 C} \text {. }
$$

The perpendicular electron heat conductivity $\kappa_{\perp e}$ is controlled by classical processes because electrons moving perpendicular to the ambient magnetic field do not feel appreciable wave-particle interactions. Similarly, ions moving parallel to the magnetic field experience very few wave-particle resonances. Hence, $\kappa_{\mid j i}$ also retains its classical value. The perpendicular ion heat conductivity is found from the usual expression which relates the heat conductivity to spatial diffusion,

$$
\kappa_{\perp i}{ }^{I C}=n_{e} k_{B} D_{\perp}^{* i}
$$

where $k_{B}$ is Boltzman's constant. From equations (6) and (14) the expression for $\kappa_{\perp i}{ }^{\text {IC }}$ reduces to

$$
\kappa_{i i}{ }^{I C} \simeq\left(e / m_{i}\right)^{2}\left(n_{e}^{2} k_{B} T_{e} / \Omega_{i}^{3}\right) \epsilon_{s} .
$$

In a non-turbulent plasma, thermal transport perpendicular to the ambient magnetic field is smaller than that parallel to the field. This is because the cyclotron motion ties particles to the field lines. However, in a turbulent plasma the electric field fluctuations knock particles out of their cyclotron trajectories thereby destroying the constraining effects of the magnetic field. As a result, $\kappa_{1 . i}{ }^{I C}$ is larger than its classical counterpart.

The total energy which is given to the electrons is $\mathbf{j} \cdot \mathbf{E}$, where $\mathbf{j}$ is the current density and where $\mathbf{E}$ is the electric field which drives this current. Since $\mathbf{E}=\eta^{x \boldsymbol{c}} \mathbf{j}$ we therefore see that the electrons are given energy at the Joule rate, i.e.

$$
Q_{e}^{\text {Joule }}=\eta^{\text {IC }} i^{2} .
$$

Some of this energy, however goes into electrostatic ion cyclotron waves which subsequently heat the ions. Ions are heated primarily in a direction which is perpendicular to the ambient magnetic field (Dakin et al, 1976). Taking the second velocity moment of equation (1) indicates that ions which are perpendicular to the field heat at a rate which is 
given by

$$
Q_{\perp i}^{\text {wave-ion }} \simeq n_{i} m_{i} D_{\perp}{ }^{i}
$$

where $n_{i}$ is the ions number density and where $D_{\perp}^{\prime}$ is given by equation (14).

In order that we may apply these transport coefficients (equations (15), (16) and (19)) and heating rates (equations (20) and (21)) to the topside ionosphere, we must determine the saturated level of turbulence $\epsilon_{\mathrm{s}}$. The saturation occurs in two stages and is discussed by lonson et al. (1976). The first stage saturation level is reached after a few Larmor periods and lasts only seconds with the subsequent development of a second stage level. This second stage is practically steady, possessing low level fluctuations and lasts throughout the length of the disturbance. In the following sections a more detailed discussion of these two stages is presented.

\section{FTRST STAGE LEVEL OF IC TURBULENCE}

Assuming that the instability threshold for electrostatic ion-cyclotron waves is cxcceded, Dum and Dupree (1970) investigated a first stage saturation process. The saturated spectrum results from a nonlinear damping of the waves by thermal ions. Saturation occurs when the number of absorbing ions becomes sufficiently large to balance the stimulated emission of waves. Since the number of absorbing ions is related to the ion resonance width $\left(\Delta \omega_{\perp}\right)_{i}$, the instability saturates when the energy density of the turbulence reaches a value which forces the following equality to be satisfied:

$$
\left(\Delta \omega_{\perp}\right)_{i}=\gamma_{L}
$$

where $\gamma_{L}$ is the linear growth rate of the instability.

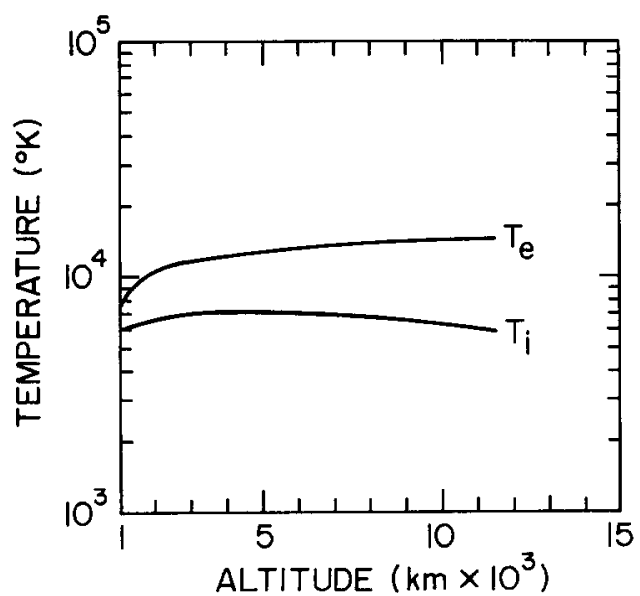

Fig. 1. TYPiCAl STEADY STATE ION AND ElEctron TEMPERATURE PORFILES DURING AN AURORAL EVENT.

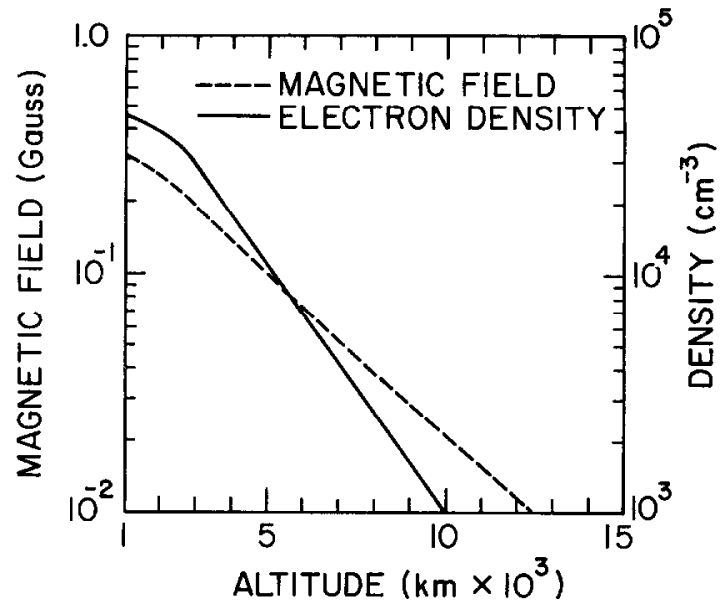

Fig. 2. TyPiCAl electron density and Magnetic field STRENGTH DURING AN AURORAL DISTURBANCE.

The value of $\gamma_{L}$ is typically given by

$$
\gamma_{L} \simeq 10^{-2} \Omega_{i}
$$

Using equations (9), (14), (22) and (23), the first stage level of the instability saturates at an energy density which is given by

$$
\epsilon_{s}^{1 s t} \simeq(0.1)\left(\Omega_{i} / \omega_{p i}\right)^{2} .
$$

We have calculated the electrical resistivity (equation (15)), electron and ion thermal conductivities (equations (16), (17) and (19)) as well as the turbulent heating rates (equations (20) and (21)) based upon the value $\epsilon_{s}^{\text {1st }}$ given in equation (24). The various plasma parameters which were incorporated into these calculations were obtained from an ionosphere model (including both $\mathrm{O}^{+}$and $\mathrm{H}^{+}$) pertaining to conditions during the magnetic storm of August 4, 1972 (see Figs. 1 and 2) (Fontheim $e t$ al., 1978). In Fig. 3 we plot the anomaly factor (i.e. ratio of anomalous to classical transport coefficients) of the resistivity and thermal conductivity as functions of altitude for typical values of current density. In Fig. 4 we display the rate of turbulent heating as a function of altitude and current density. From Fig. 4 it is to be observed that the first stage perpendicular heating of the ions takes place at a rate of about $10^{-8} \mathrm{ergs} \mathrm{cm}^{-3} \mathrm{~s}$. As a result $T_{i}^{\perp} / T_{i}^{\|}$could increase by a factor of ten in only a few seconds. However, we will see in the next section that a second stage of the saturation process limits the magnitude of the ion temperature anisotropy. 


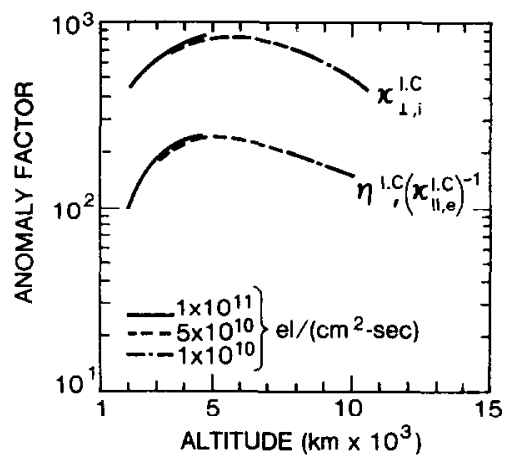

Fig. 3. First stage anomalous transport COEFFCIENTS, ELECTRON (ION) PARALLEL (PERPENDICULAR) HEAT CONDUCTION AND THE RESISTIVITY NORMALIZED TO THEIR CLASSICAL VALUES FOR THREE VALUES OF CONSTANT CURRENT DENSITY.

(Note: $1 \mathrm{el} \mathrm{cm}^{-2} \mathrm{~s}=1.6 \times 10^{-15} \mathrm{~A} \mathrm{~m}^{-2}$ ).

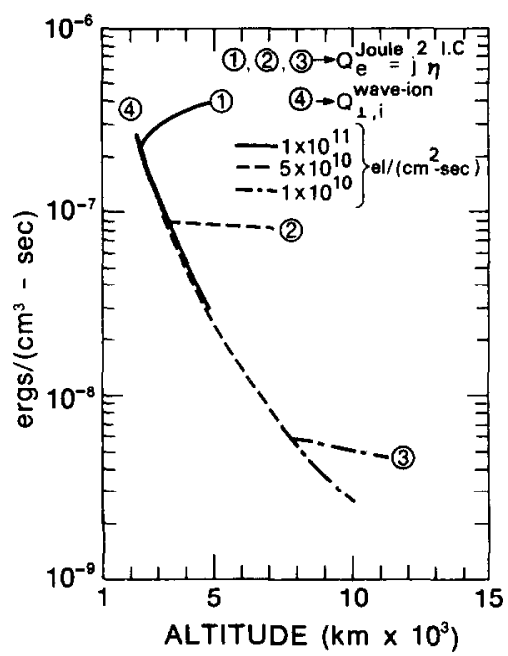

Fig. 4. CuRves 1, 2 AND 3 Represent the first StAGe ANOMALOUS JOULE HEATING OF THE ELECTRONS FOR THREE VALUES OF CONSTANT CURRENT DENSTIYY.

Curve 4 represents perpendicular heating (wave-ion) of the ions for the same three values of current density.

\section{SECOND STAGE LEVEL OF TURBULENCE}

Lee (1972) has studied the effect of thermal anisotropies on the electrostatic ion cyclotron instability and found that an increase in $T_{i}^{\perp} / T_{i}^{\|}$raises the instability threshold (Fig. 5). Therefore, the insta- bility threshold increases as a consequence of first stage heating (leading to an increase in $T_{i}^{\perp} / T_{i}^{\|}$) until some critical value $\left(T_{i}^{\perp} / T_{i}^{\|}\right)_{\text {critical }}$ is reached (Fig. 6). At this critical value of the ion temperature anisotropy, the threshold has increased to the point where electrostatic ion-cyclotron waves are no longer destabilized, i.e. the instability shuts off. However, a residual second stage level of IC turbulence remains which is the result of a balance between processes which tend to increase $T_{i}^{\perp} / T_{i}^{\| \prime}$ (heating of ions by second stage turbulence) and processes which tend to destroy ion temperature anisotropy (ion-ion collisions). For typical ionospheric parameters this second stage level of turbulence is reached in only a few seconds.

We now estimate the second stage level of IC turbulence. Assuming that ion-ion collisions play the role of restoring ion temperature isotropy, we have

$$
\left.\frac{\partial}{\partial t}\left(T_{i}^{\perp}-T_{i}^{l}\right)\right|_{\text {ion-ion }} \simeq-v_{i i}\left(T_{i}^{\perp}-T_{i}^{\|}\right)
$$

where $v_{i i}$ is the ion-ion collision frequency. From equations (14) and (21) we can also write

$$
\left.\frac{\partial}{\partial t}\left(T_{i}^{\perp}-T_{i}^{\|}\right)\right|_{\text {wave-ion }}=\left(e^{2} / m_{i}\right)\left(n_{e} T_{e} / \Omega_{i}\right) \epsilon_{s}^{2 n d} .
$$

Equating equations (25) and (26) yields an expression for the second stage level of turbulence which is given by

$$
\epsilon_{\mathrm{s}}^{2 \mathrm{nd}} \simeq\left(m_{i} / e^{2}\right)\left(\Omega_{i} / n_{e} T_{e}\right)\left(T_{i}^{\perp}-T_{i}^{\|}\right) v_{i i}<\epsilon_{s}^{1 \text { st }} .
$$

Using the second stage energy density described by equation (27) in equations (15), (16) and (19) through (21) we have calculated the anomaly factor (i.e. ratio of anomalous to classical transport coefficients) of the resistivity and thermal conductivity as functions of altitude for typical values of current density. These results are displayed in Fig. 7 and indicate that transport processes are somewhat enhanced above their classical analogues. In Fig. 8 we show the second stage rate of turbulent electron and ion heating. This heating is practically steady and lasts throughout the length of the disturbance.

In a recent paper the observations of Hudson et al. (1978) suggest that the second stage level of turbulence is not reached. However, the event they discussed does not necessarily characterize all possible auroral conditions. Whether or not the second stage level of IC turbulence is reached depends upon how the system evolves. This would require additional measurements as well as a more refined analysis of the process. The latter must involve a 


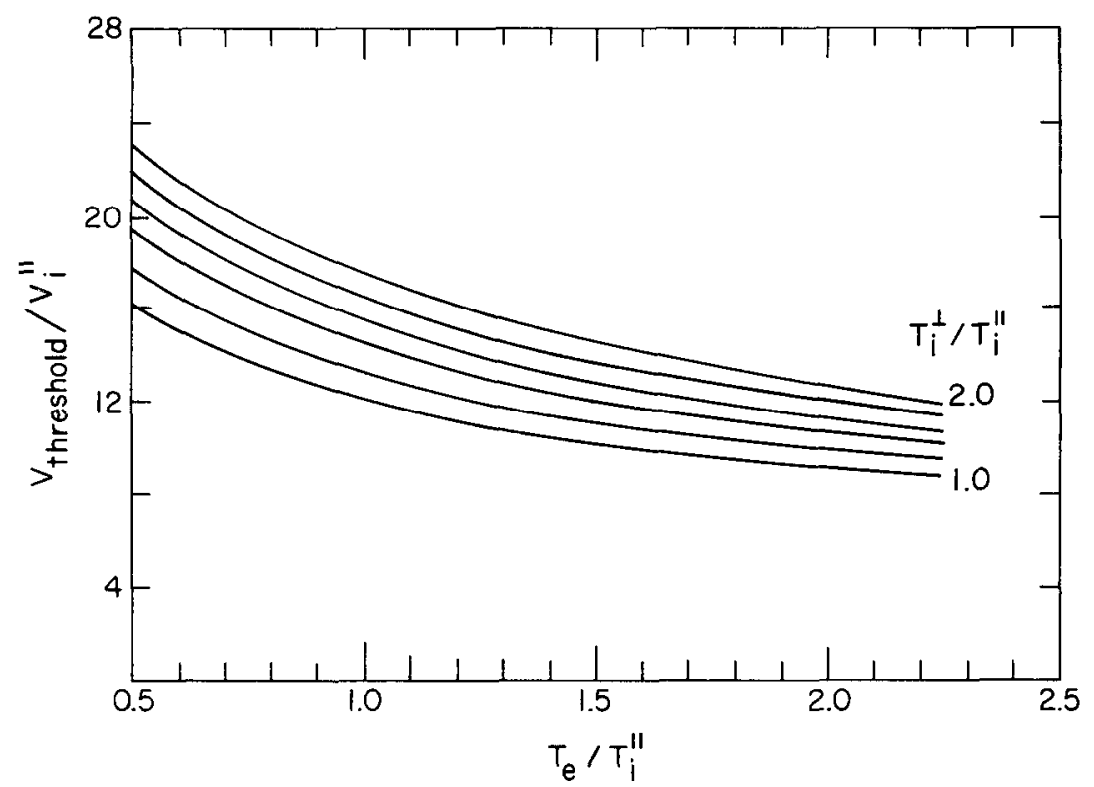

Fig. 5. THReshold electron DRIFT NORMALIZED to THE PARALlel ION THERMal Velocity VS THE RATIO OF ELECTRON TO PARALLEL ION TEMPERATURES FOR DIFFERENT VALUES OF THE RATIO $T_{i}^{\perp} / T_{i}^{\| \prime}$.

Note that as the second stage proceeds, the critical velocity is described by progressively higher contours while $T_{e} / T_{i}^{\|}$remains approximately constant.

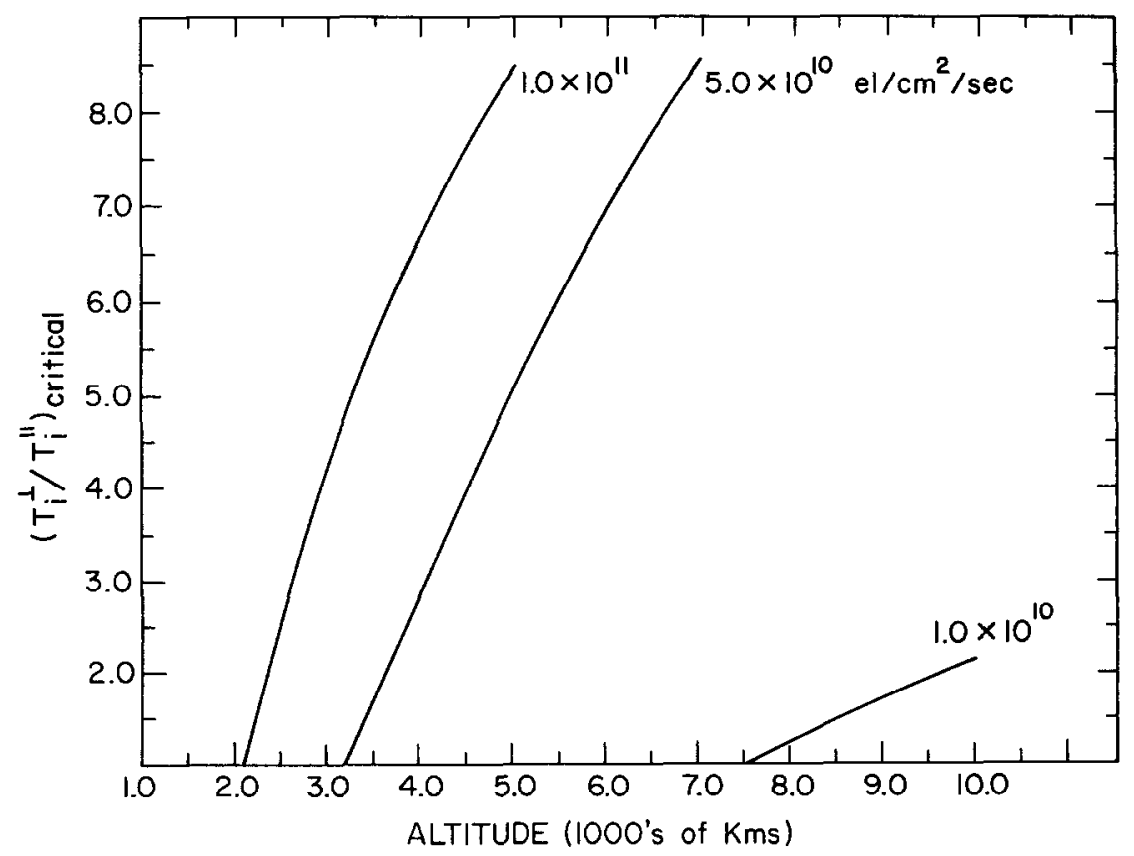

Fig. 6. Critical ION temperature anisotropy vs altitude using plasma parameters From a TYPICAL IONOSPHERIC ENERGY BALANCE MODEL.

For a particular constant current density (represented by the curves in units of el. $\mathrm{cm}^{-2} \mathrm{~s}^{-1}$ ) first stage turbulence will raise $T_{i}^{\perp}$ until $\left(T_{i}^{\perp} / T_{i}^{l \mid}\right)=\left(T_{i}{ }^{\perp} / T_{i}^{l /}\right)_{\text {critical }}$. 


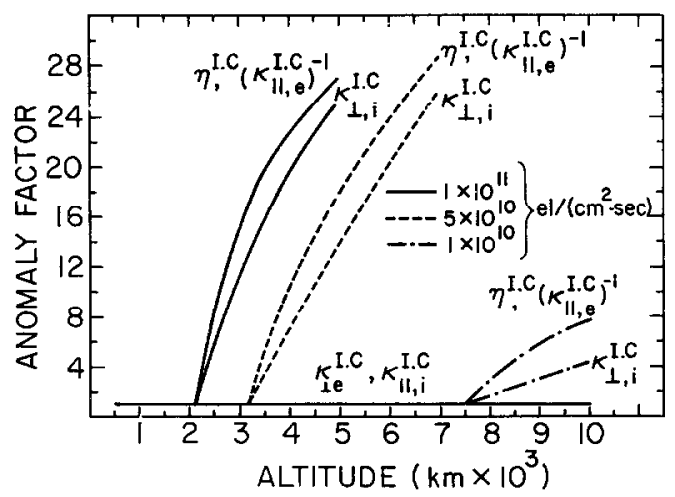

Fig. 7. Second stage anomalous transport coeffiCIENTS, ELECTRON (ION) PARALLEL (PERPENDICULAR) HEAT CONDUCTIVITIES AND RESISTIVITY NORMALIZED TO THEIR CLASSICAL VALUES FOR THREE CONTOURS OF CONSTANT CURRENT DENSTTY.

As mentioned in the text $\kappa_{\perp e}$ and $\kappa_{\| i}$ retain their classical values.

complete energy balance which takes into consideration the changing conditions as the plasma is heated.

\section{CONCLUSION}

Saturation of the electrostatic ion cyclotron instability is shown to proceed in two stages. The first stage is essentially the result of Dum and Dupree (1970) and lasts only a few seconds. This stage is characterized by a substantial heating of the ions perpendicular to the background magnetic field (geomagnetic field) and is so large that for all practical purposes $T_{i}^{\|}$and $T_{e}$ can be held fixed compared to $T_{i}^{\perp}$. After a few seconds $T_{i}^{\perp} / T_{i}^{\mathrm{il}}$ reaches a critical value after which the instability almost shuts off, leaving a steady state second stage level of ion cyclotron turbulence. At this second stage level the tendency for ion-ion collisions to restore ion temperature isotropy is balanced by the generation of anisotropy through turbulent heating of the ions.

Using the second stage level of electrostatic ion cyclotron turbulence and plasma parameters from a stormtime ionosphere energy balance model, we have calculated the electrical resistivity, electron and ion thermal conductivities and the rate of turbulent heating for typical current densities. These results are given as a function of altitude (Figs. 7 and 8) and indicate an anomalous increase in field-aligned electrical resistivity and cross-field ion thermal conductivity as well as a decrease in electron thermal conductivity along the geomagne-

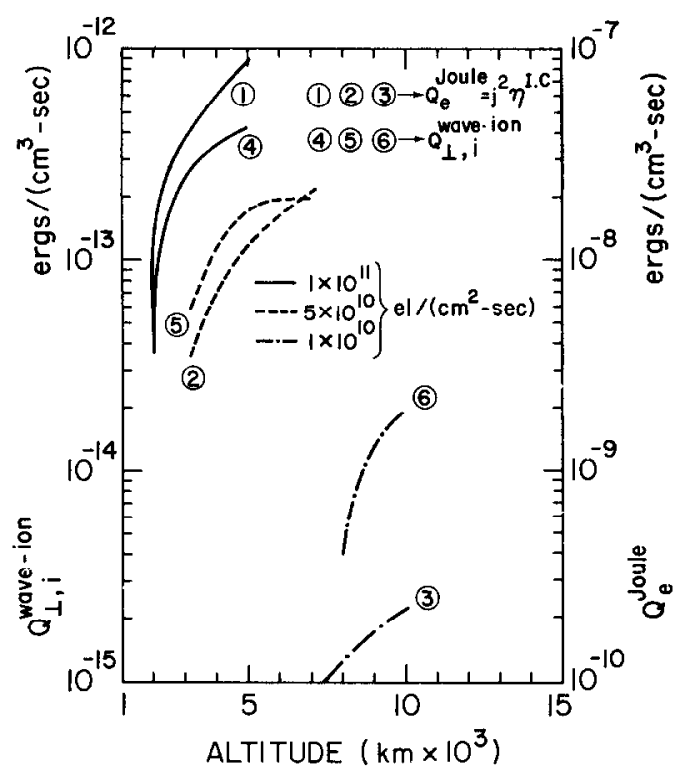

Fig. 8. Curvis 1, 2, And 3 represent ti ie second stage ANOMALOUS JOULE HEATING OF THE ELECTRONS FOR THREE VALUES OF CONSTANT CURRENT DENSITY.

Curves 4, 5 and 6 represent the second stage perpendicular heating (wave-ion) of the ions for the same three values of current density.

tic field. In addition, turbulent heating leads to a significant ion temperature anisotropy (Fig. 6). These conditions exist for the duration of an auroral disturbance.

Acknowledgments--This work was supported by NSF Grant ATM 73-00322 A01 and NASA Grant NGR 23005-015.

\section{REFERENCES}

Arnoldy, R. L. (1978). Rev. geophys. Res. 12, 217.

Biskamp, D. and Chodura, K. (1971). 4th Int. Conf. Plasma Phys. \& Controlled Nuc. Fusion Res., Madison, Wisconsin.

Capone, M. Z. and Davidson, R. C. (1973). Phys. Fluids $31,86$.

Cloutier, P. A., Anderson, H. R., Park, R. J., Vondrak, R. R., Spiger, R. J. and Sandel, B. R. (1970). J. geophys. Res. 75, 2595.

Dakin, D. R., Tajima, T., Benford, G. and Rynn, N. (1976). J. Plasma Phys. 15, 175.

Drummond, W. E. and Rosenbluth, M. N. (1962). Phys. Fluids, 5, 1507.

Dum, C. T. and Dupree, T. D. (1970). Phys. Fluids 13, 2064.

Fontheim, E. G., Ong, R. S. B., Roble, R. G., Mayr, H. G., Baron, M. J., Hoegy, W. H., Wickwar, V. B., Vondrak, R. R. and Ionson, J. A. (1978). J. geophys. Res. In press. 
Gary, P. S. and Paul, J. W. M. (1971). Phys. Rev. Lett. 26, 1097.

Hudson, M. K., Lysak, R. L. and Mozer, F. S. (1978). Geophys. Res. Lett. 5, 143.

Ichimaru, D. J. (1975). Phys. Soc. Japan 39, 261.

Ionson, J. A., Ong, R. S. B. and Fontheim, E. G. (1976). Geophys. Res. Lett. 3, 549.

Kindel, J. M. and Kennel, C. F. (1971). J. geophys. Res. 76, 3055 .
Lee, K. F. (1972). J. Plasma Phys. 8, 379.

Mozer, F. S. (1976). Ann. Geophys. 32, 97.

Reasoner, D. L. and Chappell, C. R. (1973). J. geophys. Res. 78, 2176.

Spitzer, L. (1962). Physics of Fully Ionized Gases, p. 120. Interscience, New York.

Weinstock, J. (1970). Phys. Fluids 13, 2308.

Weinstock, J. (1972). Phys. Fluids 15, 454. 